\title{
DEATH OF OSTEOCYTES
}

\author{
ELECTRON MICROSCOPY AFTER IN VITRO ISCHAEMIA
}

\author{
J. JAMES, G. L. STEIJN-MYAGKAYA
}

\author{
From the Academic Medical Centre, University of Amsterdam
}

\begin{abstract}
Ischaemia kills osteocytes, but opinions differ as to how long they can survive. These differences are due to the varying methods of inducing ischaemia, and to the different criteria for diagnosing cell death.

Using rabbit bone and a technique of in vitro ischaemia at $37 \mathrm{C}$, we have shown by electron microscopy that, after up to two hours, the changes which occur are probably reversible; after four hours, the cells were irreversibly damaged. This difference could not be detected by light microscopy. After 24 hours of ischaemia, most lacunae were empty or contained only osteocyte debris. We conclude that osteocytes suffer irreversible damage after in vitro ischaemia of about two hours, which is much the same response as that of most other mammalian cells.
\end{abstract}

The death of osteocytes, a point of concern in bone surgery, is often due to ischaemia, although radiation damage (Dambrain and Dhem 1984), infection, or other factors (Arlot et al. 1983) may have a role. Although bone containing dead cells may fulfil its mechanical function for some time without gross failure (Glimcher and Kenzora 1978), it does not survive as a tissue and is subsequently resorbed and replaced by fibrous tissue, cartilage or new bone. There is still some controversy with regard to the conditions which may lead to the ischaemic death of osteocytes.

Some authors consider that osteocytes can survive complete ischaemia for 48 hours (Kenzora et al. 1978), five days (Brown and Cruess 1982) or even longer (Abbott et al. 1947), though Rösingh and James (1969) claim that the period of survival is less than six hours and Kuwata. Kawai and Doi (1984) mention a period of less than 12 hours. The criteria used to determine osteocyte death by light microscopy differ widely. The methods of inducing ischaemia also vary and include ligature of afferent vessels (Abbott et al. 1947; Rösingh and James 1969) and bone transplantation (Kenzora et al. 1978; Albrektsson 1982); with either method it may be that oxygen and nutrients still reach superficial parts of the tissue. It has been estimated that up to a depth of $0.3 \mathrm{~mm}$ some osteocytes in their lacunae may survive autotrans-

J. James, MD. Professor of Histology

G. L. Steijn-Myagkaya. MD. Senior Scientist

Laboratory of Histology and Cell Biology. University of Amsterdam. Academic Medical Centre. Meibergdreef 15, 1105 AZ Amsterdam. The Netherlands.

Requests for reprints should be sent to Professor J. James.

( 1986 British Editorial Socicty of Bone and Joint Surgery $0301-620 \times 864134 \$ 2.00$ plantation without blood supply (Brown and Cruess 1982). Local variations in the degree of ischaemia, resulting in irregular patterns of necrosis, are also a problem in the study of necrosis after ligating or clamping vessels.

We have adapted an in vitro model of ischaemia to study total ischaemia in part of an organ. This method, involving the air-tight wrapping of a tissue specimen and incubation at $37 \mathrm{C}$, gives a uniform pattern of cellular decay for comparable cell types (Chang et al. 1958; Majno, La Gattuta and Thompson 1960). Similar degradative processes take place with ischaemia in vivo or in vitro. These processes can be modified by external factors such as temperature (Trump, Berezesky and Cowley 1982) and they take place a little more slowly in vitro than in vivo (Jennings, Ganote and Reimer 1975).

In our exploratory investigation, bone fragments from the rabbit femur have been studied with light and electron microscopy after different periods of in vitro ischaemia, in order to establish data with regard to the moment of actual death of the osteocytes.

\section{MATERIAL AND METHODS}

Freshly excised 1 to $2 \mathrm{~mm}^{3}$ fragments from the head and shaft of the femur of one-year-old New Zealand white male rabbits were wrapped in plastic draping material (Barrier Surgikos, Johnson and Johnson Company, New Brunswick, USA) and placed in an incubator at $37^{\circ} \mathrm{C}$ for differing periods of 15 minutes, 1, 2, 4 and 24 hours.

After incubation each fragment was unwrapped and fixed, together with fresh control fragments, in a mixture of $4 \%$ paraformaldehyde and $1 \%$ glutaraldehyde in $0.15 \mathrm{M}$ phosphate buffer $(\mathrm{pH} 7.4)$ for three days at $4 \mathrm{C}$. After fixation the fragments were demineralised at $4 \mathrm{C}$ for 14 days in $0.1 \mathrm{M}$ EDTA in $0.06 \mathrm{M}$ cacodylate buffer 

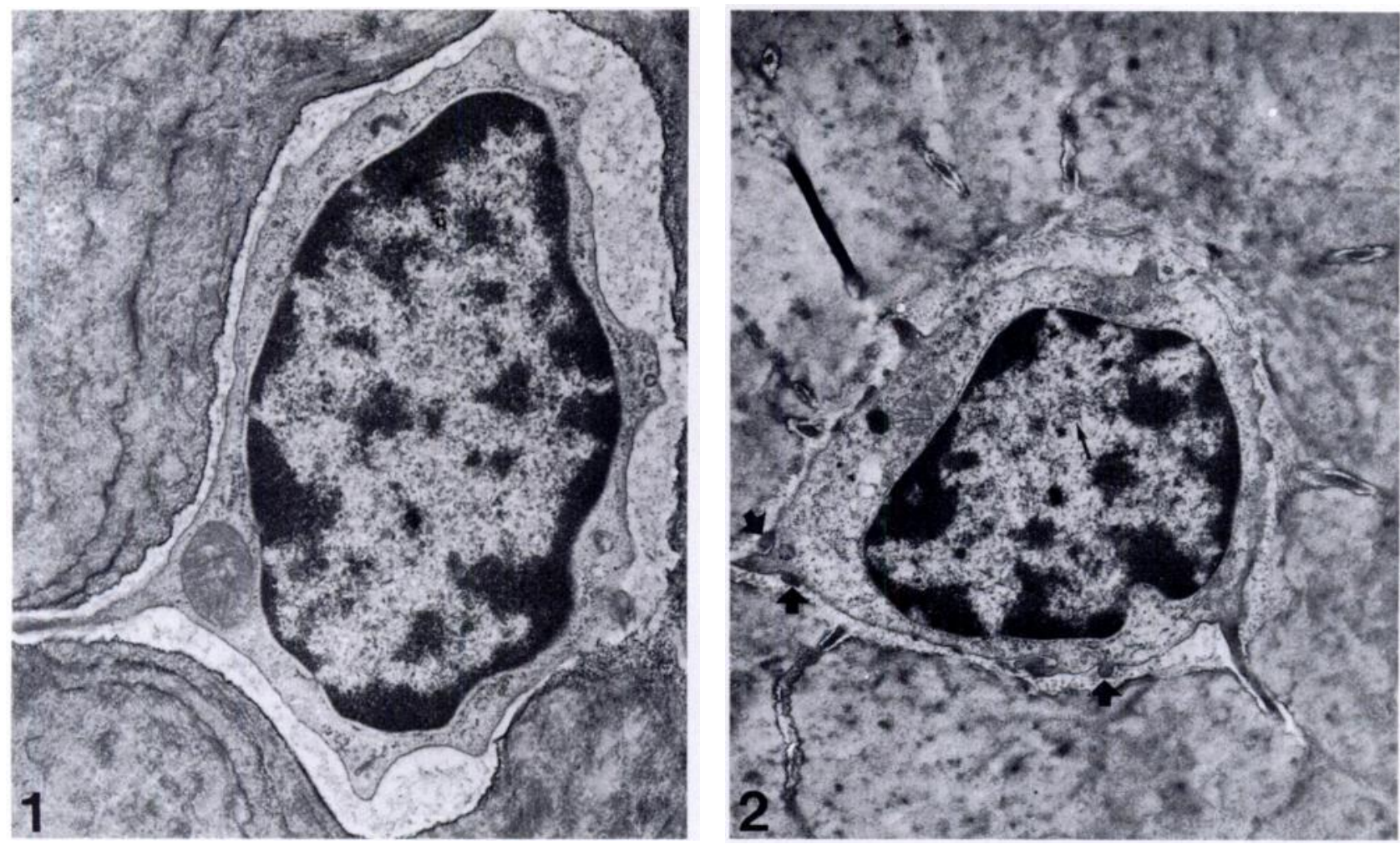

Figure 1 - A normal osteocyte in its lacuna. Note the smooth outline of the lacunar border $(\times 15000)$. Figure $2-$ An osteocyte after two hours in vitro ischaemia at $37 \mathrm{C}$ showing aggregation of the ribosomes and blebbing of the cytoplasm (thick arrows). Note segregation of interchromatin granules from the chromatin (arrow) $(\times 10000)$.

containing $2.5 \%$ glutaraldehyde (final $\mathrm{pH} 7.4$ ). After post-fixation in $1 \% \mathrm{OsO}_{4}$ in $0.1 \mathrm{M}$ cacodylate buffer for one hour at $4 \mathrm{C}$, the specimens were dehydrated through increasing strengths of ethanol and embedded in LX 112 (Ladd Industries, Burlington, USA).

Semi-thin $1 \mu \mathrm{m}$ sections were cut with glass knives and stained with methylene blue. Ultra-thin sections were cut with a diamond knife, stained with uranyl acetate and lead citrate and examined with a Philips EM 300 electron microscope. For each period of incubation at least 30 fully-cut osteocytes were studied from both ischaemic and control specimens.

\section{RESULTS}

\section{Electron microscopy}

Control specimens. Osteocytes from rabbit femora showed the well-known features of osteocytes in general (Vaughan 1970; Holtrop 1975; Ham and Cormack 1979). When cells located 100 to $200 \mu \mathrm{m}$ or more from the bone surface were studied, there was no difference in shape, aspect or arrangement between the osteocytes from the femoral head and those from the shaft of the femur. The oval nucleus occupied the largest part of the cell body with a comparatively narrow rim of cytoplasm surrounding it. The nucleus showed finely granular chromatin condensed in a cap-like layer on the inner side of the nuclear envelope (Fig. 1). The majority of (poly)ribo- somes were of the free type; the rough endoplasmic reticulum was represented by a few scattered profiles of cisternae; the Golgi complex also developed. There were a few mitochondria and a few scattered lysosomes. The lenticular lacuna was sharply delineated.

In vitro ischaemia. After 15 minutes' incubation no alterations were seen. No differences could be seen between fragments incubated for one or for two hours. Both showed differences from the control material consisting of irregularity of the cellular surface (Fig. 2, thick arrows) and a tendency to the aggregation of free ribosomes. Local enlargement of the perinuclear cisternae was observed, while in the nucleus enlarged condensations of chromatin were found against the nuclear envelope. This is not shown in Figure 2. In the euchromatic parts of the nucleus, interchromatin granules appear to become separated from the chromatin: they form clusters in the widened interchromatin space (Fig. 2, arrow).

Four hours' incubation. After four hours the osteocytes show local interruptions in the continuity of the plasma membrane, which has disappeared totally over some areas of the cytoplasm. There are local extrusions of cytoplasmic content and vesiculation of the cytoplasmic matrix (Figs 3 to 5). The area of the lacuna occupied by the osteocyte has become much smaller. The nuclei show focal dilatations of the perinuclear cisternae and a condensation of the chromatin into large aggregates; in the interchromatin spaces aggregates of interchromatin 

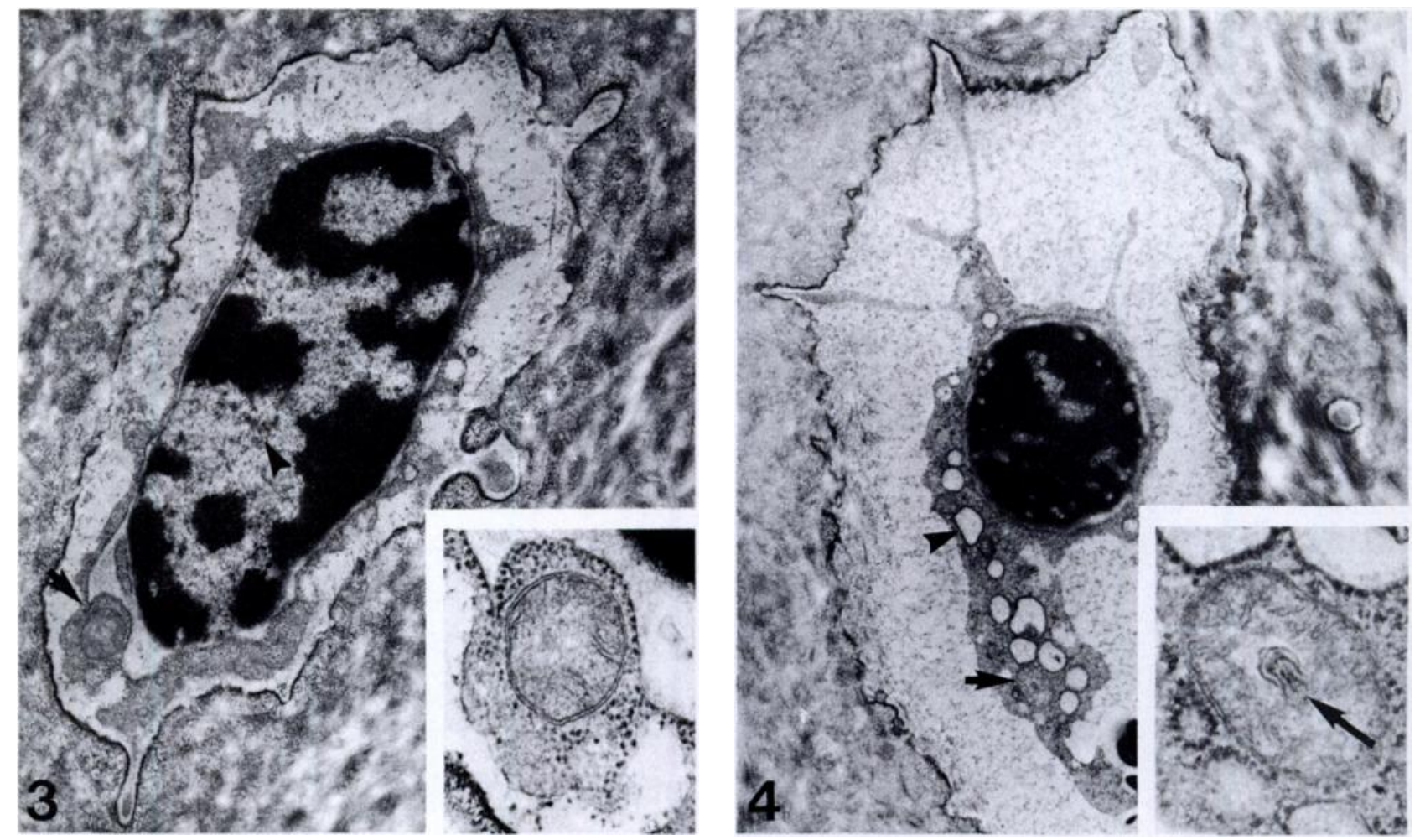

Osteocytes after four hours of ischaemia. Figure 3 - Osteocyte showing disruption of the plasma membrane with extrusion of cytoplasmic content. Note the clumping of chromatin and the aggregation of interchromatin granules (arrowhead) $(\times 12000)$. Inset: mitochondrion showing disorganisation of crista profiles $(\times 33000)$. Figure 4 - Osteocyte with disrupted plasma membrane, displaying vesiculation of the rough endoplasmic reticulum (arrowhead) $(\times 10000)$. Inset: mitochondrion showing membraneous whorl (arrow) $(\times 52000)$.

granules can be found (Fig. 3, arrowhead).

The mitochondria show no flocculent densities but they display a disorganisation of the cristae and lack of homogeneity of the matrix, sometimes combined with the appearance of membraneous whorls in the inner compartment (Figs 3 and 4 insets). Cisternae of rough endoplasmic reticulum, which are inconspicuous in normal osteocytes (Fig. 1) or after one or two hours' ischaemia (Fig. 2), have become swollen and so are more striking (Figs 4 and 5, arrowheads). The matrix lining of the lacunae show irregularities, with some protrusions into the lumen (Fig. 5, arrow).

After 24 hours' incubation. At this stage, virtually all lacunae are empty or contain only electron-dense, unidentifiable necrotic remnants of osteocytes, most of which are attached to the lacunar wall (Fig. 6).

\section{Light microscopy}

Under the light microscope, in $1 \mu \mathrm{m}$ sections stained with methylene blue, osteocytes after two hours' incubation showed a considerably more dense nucleus than in control specimens with a pyknotic appearance (Figs 7 and 8). No notable difference, however, could be seen between osteocytes after two hours' and four hours' incubation (Figs 8 and 9). After 24 hours' incubation there was a dramatic difference: virtually all lacunae seemed empty and there was no trace of blood vessels in the haversian canals (Fig. 10).

\section{DISCUSSION}

After four hours of in vitro ischaemia the majority of osteocytes are so damaged that they have to be considered to be beyond the "point of no return" (Stages V to VI, Trump et al. 1980). While the changes seen after two hours, such as condensation of the nuclear chromatin into large clumps (Fig. 2) and irregularities of the surface due to disorganisation of the cytoskeleton, are known to belong to the reversible phase of ischaemia (Lemasters et al. 1982; Myagkaya, van Veen and James 1984), those seen after four hours' incubation definitely go beyond this. Coarse aggregation of chromatin, with broadening of the interchromatin space and aggregation of interchromatin granules, is combined in the latter stage with the appearance of large interruptions on the plasma membrane and extrusion of parts of the cytoplasmic content.

Flocculent densities in the mitochondrial matrix, often considered to be a sign of irreversible cell damage (Trump et al. 1982), have not been observed in the mitochondria of ischaemic osteocytes, but it has been shown that this sign is not always reliable (Myagkaya et al. 1984). An indication of disorganisation of mitochondrial structure which we did see in the four-hour specimens was the appearance of membraneous whorls within mitochondria which often showed deformation of their matrix structure (Fig. 4, inset). 

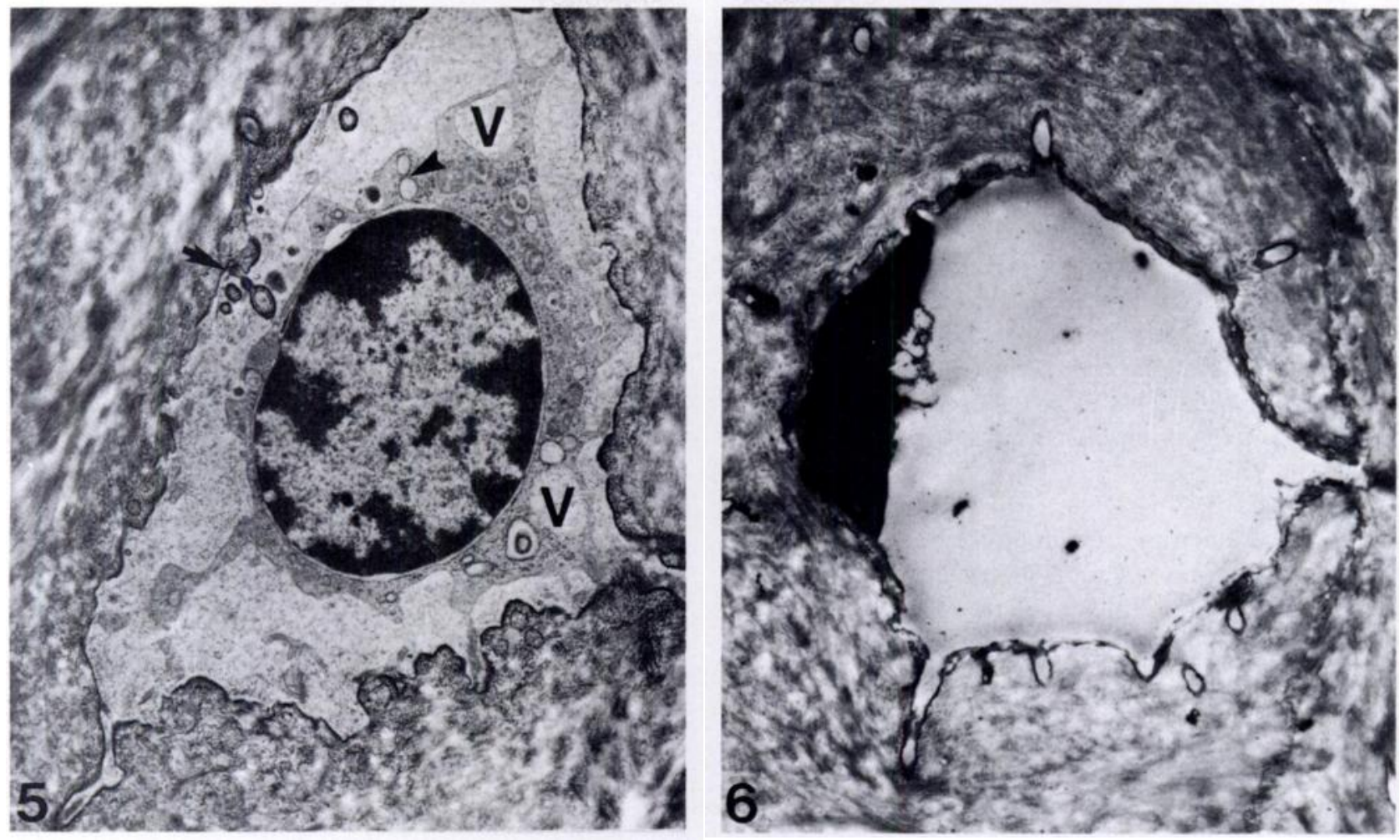

Figure 5 An osteocyte after four hours of ischaemia, showing disrupted plasma membrane, extrusion of cytoplasmic content and vesiculation of the endoplasmic reticulum as in Figures 3 and 4 (arrowhead) with, in addition, large vacuoles $(V)$ in its cytoplasm. Note the irregularity of the lacunar border. which is a phenomenon often seen around necrotic osteocytes $(\times 10000)$. Figure 6 - Necrotic remnants of an osteocyte as seen after 24 hours of in vitro ischaemia $(\times 9000)$.
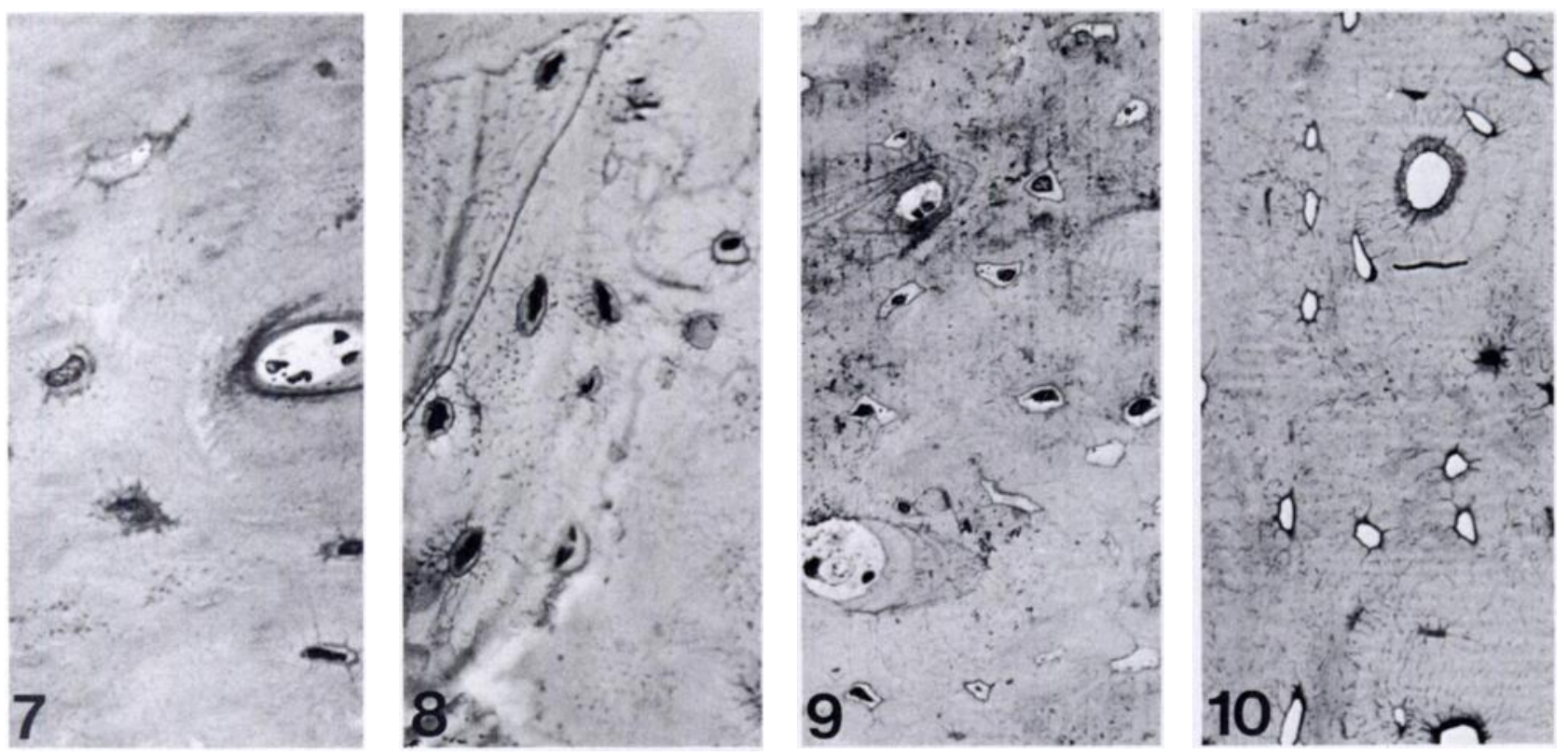

Figures 7 to 10 - Light micrographs of fragments of rabbit bone after various periods of in vitro ischaemia (methylene blue $\times 800$ ). Figure 7 Control specimen. Figure 8 After two hours' ischaemia. Figure 9 - After four hours' ischaemia. Figure 10 - After 24 hours' ischaemia. Further explanation is in the text. 
Although the signs of mitochondrial deterioration may not be considered conclusive, interruptions of the plasma membrane and disorganisation of the cytoplasm are definite signs of irreversible damage which were seen in a large majority of osteocytes following four hours of in vitro ischaemia. Definite proof of non-viability could only have been provided by testing after restoration of the circulation, which is impossible with this model.

The differences between osteocytes submitted to in vitro ischaemia for two hours and for four hours cannot be detected by light microscopy. Since the cytoplasm of an osteocyte is seen only as a rim around the nucleus under these conditions, the interpretation has to be based on nuclear shape and size, with an impression of the nuclear chromatin. This means that the actual translation into an irreversible phase cannot be detected with the light microscope.

Degradation of DNA in osteocytes following ischaemia, a later sign of cellular decay, can be shown histochemically only six hours after clumping of afferent vessels in the rabbit femoral head (Rösingh and James 1969). The total disappearance of osteocytes from the lacunae which has been often used as a criterion for osteocyte damage (Dambrain and Dhem 1984) is again a later sign; we observed it generally under light microscopy after 24 hours of in vitro incubation (Fig. 10). Empty lacunae could be the result of loss of necrotic osteocyte debris in the making or processing of sections. It is also likely that the situation shown in Figure 6 would be interpreted as an empty lacuna under light microscopy.

Concerning the original question of the viability of osteocytes under total ischaemia, our observations provide good arguments for the assumption that osteocytes can tolerate no more than two hours of total ischaemia. If this is so, they behave like all other nucleated human cells, which in general can endure in vivo ischaemia at $37 \mathrm{C}$ (temperature is of course very important) for a maximum of two hours before sustaining irreversible damage (Majno et al. 1960). There are no sound arguments for the extension of this period to one of several days for osteocytes (Kenzora et al. 1978; Albretksson 1982). A surgeon working with such techniques as free vascularised bone transplants (Brown and Cruess 1982) in which living osteocytes are important should reckon on a maximum period of ischaemia of two hours or even a little less, in view of the slight difference in tempo between cellular decay in vitro and in vivo (Jennings et al. 1975).
Our thanks are due to Dr J. Vegter for drawing our attention to the problem, to $\mathrm{Mr} \mathrm{H}$. van Veen for excellent technical assistance, Dr W. Frederiks and V. Everts for helpful comment, $\mathrm{Mr} \mathrm{C}$. Gravemeyer for photographic work and Mrs T. Pierik for secretarial assistance.

\section{REFERENCES}

Abbott LC, Schottstaedt ER, Saunders JBdeCM, Bost FC. The evaluation of cortical and cancellous bone grafting material: a clinical and experimental study. J Bone Joint Surg 1947;29:381-414.

Albrektsson T. Ischaemia and bone grafts. Scand J Plast Reconstr Surg 1982:Suppl 19:21-4.

Arlot ME, Bonjean M, Chavassieux PM, Meunier PJ. Bone histology in adults with aseptic necrosis: histomorphometric evaluation of iliac biopsies in seventy-seven patients. J Bone Joint Surg $[\mathrm{Am}]$ 1983; 65-A:1319-27.

Brown KLB, Cruess RL. Bone and cartilage transplantation in orthopaedic surgery: a review. J Bone Joint Surg $[\mathrm{Am}]$ 1982;64-A: $270-9$.

Chang JP, Stowell RE, Betz HE, Berenbom M. Histochemical studies of necrosis of mouse liver in vitro. AMA Arch Path 1958;65:479-87.

Dambrain R, Dhem A. Bone vitality in induced radionecrosis of the mandible estimated by osteocytic population counting. Strahlentherapie 1984; 160:39-44.

Glimcher MJ, Kenzora JE. The biology of osteonecrosis of the human femoral head and its clinical implications: an abridged communication. Clin Orthop 1978:130:47 - 50.

Ham AW, Cormack DH. Histology 8th ed. Philadelphia etc: JB Lippincott, 1979

Holtrop ME. The ultrastructure of bone. Ann Clin Lab Sci 1975:5: 264-71.

Jennings RB, Ganote CE, Reimer KA. Ischemic tissue injury. Am $J$ Pathol 1975;81:179-98.

Kenzora JE, Steele RE, Yosipovitch ZH, Glimcher MJ. Experimental osteonecrosis of the femoral head in adult rabbits. Clin Orthop 1978; 130:8 46 .

Kuwata N, Kawai S, Doi K. Clinical and experimental studies of bone union in reimplantation of digits: a preliminary report on ischemic interval. Microsurgery 1984;5:31-5.

Lemasters JJ, Stemkowski CJ, Ji S, Thurman RG. Liver structure and function in hypoxia. In: Wauquier A. Borgers M, Amery WK, eds. Protection of tissues against hypoxia. Amsterdam etc: Elsevier Biomedical Press, 1982:15-30.

Majno G, La Gattuta M, Thompson TE. Cellular death and necrosis: chemical, physical and morphologic changes in rat liver. Virchow:s $\operatorname{Arch}[A] 1960 ; 333: 421-65$.

Myagkaya G, van Veen H, James J. Ultrastructural changes in rat liver sinusoids during prolonged normothermic and hypothermic ischemia in vitro. Virchow's Arch [Cell Pathol] 1984;47:361-73.

Rösingh GE, James J. Early phases of avascular necrosis of the femoral head in rabbits. J Bone Joint Surg $[B r] 1969 ; 51-B: 165-74$.

Trump BF, McDowell EM, Arstila AU. Cellular reaction to injury. In Hill RB, La Via MF, eds. Principles of pathobiology: 3rd ed. Oxford: Oxford University Press, 1980:20 112.

Trump BF, Berezesky IK, Cowley RA. The cellular and subcellular characteristics of acute and chronic injury with emphasis on the role of calcium. In: Cowley RA, Trump BF, eds. Pathophysiology of shock, anoxia and ischemia. Baltimore: Williams \& Wilkins, 1982:6.

Vaughan JM. The physiology of bone. Oxford: Clarendon Press, 1970. 